\title{
A PRÁTICA DE ATIVIDADES FÍSICAS EM IDOSOS E SUA CORRELAÇÃO COMUM ENVELHECIMENTO SAUDÁVEL
}

\author{
THE PRACTICE OF PHYSICAL ACTIVITIES IN ELDERLY PEOPLE \\ AND THEIRCORRELATION WITH HEALTHY AGING
}

\author{
Hirisdiane Bezerra Alves ${ }^{1}$ \\ Karla Pereira Vasconcelos ${ }^{2}$ \\ Cleisla Thamires Lacerda Silva ${ }^{3}$ \\ Kalyne Araújo Bezerra ${ }^{4}$ \\ Hirisleide Bezerra Alves ${ }^{5}$
}

RESUMO: O processo de envelhecimento é caracterizado por mudanças nos hábitos e estilo de vida. A taxa de inatividade física entre os idosos chega a ser de 32, 3\%, o que é um grande desafio para a saúde pública. Objetivo: Nesse contexto, nota-se que há uma necessidade de estudos mais aprofundados acerca da importância da prática regular de atividade física pelo idoso. Assim a presente pesquisa visa descrever a prática de atividade física em idosos e a sua correlação com um envelhecimento saudável. Método: Trata-se de uma revisão bibliográfica integrativa, onde as bases de dados MEDLINE/PUBMED, LILACS, SCIELO, DOT LIB, livros e Revistas Eletrônicas de Saúde foram consultadas para o levantamento de artigos científicos. Como critérios de inclusão utilizou-se: Artigos sem restrição de língua, que estivessem disponíveis nas bases de dados no período de busca, além de estarem dispostos integralmente. Foram utilizados 41 artigos a fim de compor a presente revisão. Para a busca foram selecionados descritores de ciências da saúde: Envelhecimento. Promoção da saúde. Exercício físico. Saúde. Resultados: A prática regular de exercícios físicos em idosos melhora as funções orgânicas e cognitivas, garantindo maior independência pessoal, prevenindo e controlando

\footnotetext{
${ }^{1}$ Enfermeira pelo Centro Universitário Maurício de Nassau/Campina Grande. Pós-graduanda em Urgência, Emergência e UTI pelo Instituto Michele Sales/Campina Grande. Pós-graduanda em Obstetrícia e Neonatologia pelo Instituto Michele Sales/Campina Grande. Dianyalves06@gmail.com.

${ }^{2}$ Graduanda em Enfermagem pela Universidade Estadual da Paraíba (Campina Grande/PB). Karlapv2017@gmail.com.

${ }_{3}$ Graduanda em Enfermagem pela Universidade Estadual da Paraíba (Campina Grande/PB). cleislalacerda@gmail.com.

${ }^{4}$ Graduanda em Enfermagem pela UNIFACISA/Campina Grande/PB. Kalyneearaújo@gmail.com.

${ }^{5}$ Mestra em Genética pela Universidade Federal de Pernambuco (UFPE). Biomédica e especialista em Microbiologia Clínica pelo Centro Universitário Maurício de Nassau/Campina Grande. Hirisleidebezerra@gmail.com.
} 
doenças como diabetes, hipertensão, síndrome sarcopénica, depressão e demência, além de atuar na prevenção de agravos à saúde do idoso como quedas e fraturas. Conclusão: A prática regular de atividades físicas pelo idosoé de suma importância na prevenção, tratamento e redução de algumas doenças, sendo um fator que atua fortemente na qualidade de vida, promoção da saúde e envelhecimento saudável.

Palavras chave: Envelhecimento. Promoção da saúde. Exercício físico. Saúde.

ABSTRACT: The aging process is characterized by changes in habits and lifestyle. The rate of physical inactivity among the elderly reaches $32,3 \%$, which is a major challenge for public health. Objective: In this context, it is noted that there is a need for more in-depth studies on the importance of regular practice ofphysical activity by the elderly. Thus, the present research aims to describe the practice of physical activity in the elderly and its correlation with healthy aging. Methods: This is an integrative bibliographic review, where the MEDLINE / PUBMED, LILACS, SCIELO, DOT LIB databases, books and Electronic Health Journals were consulted for the survey of scientific articles. As inclusion criteria we used: Articles without language restriction, that were available in the databases during the search period, in addition to being fully available. 41 articles were used in order to compose the present review. Health sciences descriptors were selected for the search: Aging. Health promotion. Physical exercise. Health. Results: Regular physical exercise in the elderly improves organic and cognitive functions, ensuring greater personal independence, preventing and controlling diseases such as diabetes, hypertension, sarcopenic syndrome, depression and dementia, in addition to acting in the prevention of health problems elderly as falls and fractures. Conclusion: The regular practice of physical activities by the elderly is extremely important in the prevention, treatment and reduction of some diseases, being a factor that acts strongly in the quality of life, health promotion and healthy aging.

Keywords: Aging. Health promotion. Exercise. Health. 


\section{INTRODUÇÃO}

O significativo aumento da população idosa, nas últimas décadas, tanto nos países desenvolvidos quanto naqueles em desenvolvimento, como é o caso do Brasil, é resultado do aumento na expectativa de vida da população, em consequência da diminuição das taxas de mortalidade e de natalidade (LEITE et al., 2015). Os idosos já representam cerca de $12 \%$ da população mundial, com estimativa de duplicar esse quantitativo até 2050 (SUZMAN et al., 2015). É importante destacar que o envelhecimento populacional é uma das maiores conquistas da humanidade e também um dos grandes desafios, visto que esse fenômeno implica numa maior atenção por parte da sociedade a esta população, seja na vida ativa, social, financeira, política, profissional ou familiar (COELHO et al., 2013). E o rápido crescimento dessa população precisa de um olhar prioritário no que diz respeito às intervenções que visem o aumento da qualidade de vida dos mesmos, o que engloba a saúde física e cognitiva.

O envelhecimento populacional é um fenômeno complexo e variável, é um processo biológico de todo ser humano, que se manifesta por alterações e consequente declínio das funções orgânicas e desgastes em vários sistemas funcionais, que acontecem de forma progressiva e irreversível que ocorre caracteristicamente em função do tempo, onde não se podedefinir um ponto exato de transição (CRUZ et al., 2015). O momento em que estas transformações ocorrem e como evoluem, diferencia-se de um indivíduo para o outro. Durante esse processo, surgem alterações no organismo, como por exemplo, alterações fisiológicas, como o mau funcionamento de algumas estruturas corporais; e mecânicas, como a incapacidadede realizar movimentos básicos do corpo humano (PASCHOAL, 2004).

Por ser um processo dinâmico e progressivo, no qual há alterações morfológicas, funcionais, bioquímicas e psicológicas, o envelhecimento traz consigo a perda na capacidade de adaptação do indivíduo ao ambiente, ocasionando maior vulnerabilidade e maior incidência de doenças, as quais são predominantemente as 
doenças crônicas não transmissíveis (DCNT). As alterações do processo de envelhecimento acontecem de maneira distinta, influenciada diretamente pelo estilo de vida adotado (SPIRDUSO, 2005; FREITAS et al., 2011).

Os fatores comportamentais de risco dos idosos juntamente com as alterações naturais do envelhecimento podem levar o indivíduo a um estado de dependência para a realização das atividades básicas da vida diária, tendo consequentemente uma diminuição significativa da saúde e qualidade de vida (COELHO et al., 2013). As mudanças nos hábitos e estilo de vida pelo processo de envelhecimento, como por exemplo a falta da prática de atividades físicas, e consequente aumento do sedentarismo é bastante incidente, o que acarreta em maior predisposição a doenças. A taxa de inatividade física chega a ser de $32,3 \%$ na população com mais de 65 anos de idade e o avançar da idade vem sendo apresentado como determinante no declínio da prática de atividades físicas (KOENEMAM et al., 2011, HALLAL et al., 2012).

A inatividade física no idoso acelera a perda de força, flexibilidade, equilíbrio e resistência cardiovascular, levando ao declínio funcional, aumento e agravo dos problemas de saúde, culminando com a perda da independência (SEGUIN et al., 2012). A não prática de atividades físicas pelo idoso além de o privar de vários benefícios também poderá contribuir para a redução da sua qualidade do sono (MEDEIROS et al., 2015) e, estudos já tem comprovado que o sono tem um papel crucial para a qualidade de vida, sendo totalmente interligado à saúde física e mental (KRYGER et al., 2011).

Portanto, nota-se que a prática regular da atividade física pode contribuir de modo significativo para uma menor predisposição a doenças crônicas não transmissíveis, bem como contribuir para uma melhor qualidade de vida, sendo assim, a presente pesquisa objetivou descrever afinco a correlação da prática de atividades físicas em idosos com o envelhecimento saudável, e consequente redução de doenças. 


\section{JUSTIFICATIVA}

Segundo a Organização Mundial de Saúde (2015), a maior longevidade pode ser considerada uma história de sucesso para a humanidade, porém o processo de envelhecimentocaracteriza-se pelo comprometimento no funcionamento de todos os sistemas do organismo, afetando a saúde física e mental dos idosos (CRUZ et al., 2015), acarretando assim na modificação do perfil epidemiológico de morbimortalidade da população.

Pela modificação do perfil epidemiológico há uma sobrecarga aos serviços de saúde pelos idosos, promovendo consequências tanto sociais quanto econômicas à saúde pública, e se tal fato não for levado em conta esse marco de sucesso para a humanidade segundo a OMS, será marcado por uma população constituída de idosos dependentes e sem autonomia, devido aos problemas advindos do envelhecimento. Com isso, pode-se afirmar que os idosos são mais propensos ao acometimento por doenças, principalmente as doenças crônicas não transmissíveis. A categoria da terceira idade já vem se tornando onipresente, e essa realidade precisa ser acolhida pelos programas e políticas de saúde pública.

Com o aumento da atividade física numa população poderá ser um grande contributo para a economia local e até mesmo nacional. Uma população mais saudável levará à redução automática dos custos do Estado com a saúde pública, nomeadamente com a diminuição dos internamentos hospitalares, na observação clínica nos centros de saúde e a diminuição da comparticipação em medicamentos, como por exemplo: os antidepressivos, os ansiolíticos e osfármacos para combater a insuficiência venosa, a insónia, a osteoporose, entre outros. Portanto, acredita-se que simples medidas como a prática regular de atividade física podem influenciar de maneira significativa nesse âmbito. Com isso, os dados da presente pesquisa são de suma importância para a efetuação de programas de atividades físicas para idosos que visem uma melhoria na saúde física e cognitiva por meio do incentivo de práticas exercícios. 


\section{MÉTODO}

A presente pesquisa trata-se de uma revisão bibliográfica integrativa, onde as bases de dados MEDLINE/PUBMED, LILACS, SCIELO, DOT LIB, livros e Revistas Eletrônicas de Saúde foram consultadas para o levantamento de artigos científicos. Como critérios de inclusão utilizou-se: Artigos sem restrição de língua, que estivessem disponíveis nas bases de dados noperíodo de busca, além de estarem dispostos integralmente. Foram utilizados 41 artigos a fim de compor a presente revisão. Para a busca foram selecionados descritores de ciências da saúde: Envelhecimento. Promoção da saúde. Exercício físico. Saúde. Foi utilizado o operador booleano AND.

Para selecionar os 41 artigos e capítulos de livro houve uma análise pelo título e conteúdo, onde aqueles achados que não continham o assunto de interesse abordado de maneiradestrinchada foram descartados. Após uma ampla seleção, os artigos e livros foram sistematicamente lidos e analisados com objetivo de descrever a prática de atividade física emidosos e a sua correlação com um envelhecimento saudável.

\section{RESULTADOS}

Durante 0 processo do envelhecimento, o organismo sofre alterações fisiológicas em sua capacidade física, principalmente nos sistemas cardiovascular e musculoesquelético. Essas alterações acarretam numa menor velocidade de movimento, diminuição do fluxo sanguíneo cerebral, redução da agilidade, da coordenação, do equilíbrio, da flexibilidade, da mobilidade articular, da massa muscular e óssea (MATSUDO et al., 2000). No entanto, evidências clínicas e epidemiológicas revelam que a prática de atividades físicas quando praticadas 
de forma regular tem efeito positivo na promoção da saúde, possibilitando prevenção e minimização dosefeitos deletérios do envelhecimento (GREMEAUX et al., 2012).

Atividade física segundo Caspersen e colaboradores (1985), pode ser definida como qualquer movimento corporal produzido pelos músculos esqueléticos, que resulta em gasto energético maior do que os níveis de repouso, sendo toda atividade física planejada, estruturadae repetitiva que objetiva a melhoria e a manutenção de um ou mais componentes da aptidão física. A atividade física quando é praticada de forma regular, torna-se um fator importante parao aumento ou preservação da força e da potência muscular, manutenção da mobilidade e da vida, além de atuar na prevenção e redução das quedas e das fraturas (TAYLOR, 2004) que são bastante comuns na terceira idade. Vários problemas que são comuns entre idosos, podem ser prevenidos ou tratados com a prática regular de exercícios, um desses problemas é a osteoporose.

A osteoporose (OP) é um problema bem comum no processo de envelhecimento, sendo definida como um distúrbio osteometabólico de origem multifatorial, caracterizado pela diminuição da densidade mineral óssea com deterioração da microarquitetura, o que conduz a um maior risco de fraturas que ocorre pela diminuição da massa óssea (WHO, 1994). Os autores Thiebauld e Sprumont descrevem que "a senescência é caracterizada pela perda de tecido ósseo que fragiliza o esqueleto" (2009, p:41), levando consequentemente a osteopenia e posteriormente à osteoporose.

Porém a prática de atividade física, especialmente aquelas que envolvem sustentação dopeso, sugere um aumento da densidade mineral óssea, pois as forças mecânicas proporcionadas pelo exercício físico agem sobre os osteoblastos para formar osso novo e este adapta-se à carga mecânica através do efeito de mecanotransdução. Com isso, iniciam-se processos bioquímicos que levam a uma resposta celular no tecido ósseo o qual ativará a produção ou a reabsorção óssea (GUSMÃO et al., 2009; SCOTT et al., 2008). Um estudo realizado por Almeida no ano 2013, foi observado que para prevenção e reversão da osteoporose, a prática de exercícios físicos é fundamental para manutenção ou aumento do tecido ósseo bem como da densidade mineral óssea através do efeito de mecanotrasdução e do 
efeito hormonal. Em contrapartida, a inatividade física pode causar osteopenia e, consequentemente a osteoporose (ALMEIDA, 2013).

Um outro problema comum ao envelhecimento é a depressão que atinge cerca de 350 milhões de pessoas no mundo, levando ao aparecimento de várias doenças, acarretando na diminuição da qualidade de vida, sendo um problema na saúde pública (SILVA et al., 2018). Adepressão é caracterizada como um distúrbio afetivo ou de humor, de natureza multifatorial, que envolve aspectos biológicos, psicológicos e sociais (GORDILHO, 2002). Os sintomas depressivos compreendem alterações que acontecem de forma frequente na população idosa, porém a atividade física apresenta um efeito benéfico em idosos deprimidos, pois atua com umasérie de fatores como qualidade do humor, respostas fisiológicas diminuídas ao estresse, imagem corporal melhorada, melhor função cognitiva e autoestima, além de melhor qualidade do sono e das atividades funcionais exigidas na vida cotidiana (KRITZSILVERSTEIN et al., 2001). Para a Organização Mundial de Saúde (OMS) a participação regular em atividades físicas leves e moderadas pode retardar os declínios funcionais. Assim, uma vida ativa melhora a saúde mental e contribui na gerência de desordens como a depressão e a demência. Existe evidência de que idosos fisicamente ativos apresentam menor prevalência de doenças mentais do que os não ativos (WHO, 2002), apresentando também uma redução dos riscos de demência(BENEDETTI et al., 2008).

Um estudo realizado por Benedetti e colaboradores em 2008, comprovou a associação entre níveis de atividade física e a depressão, onde os idosos ativos apresentaram menor frequência de depressão. Segundo Coelho et al (2014).

Os mecanismos neurofisiológicos da atividade física que podem beneficiar a saúde mental incluem: a liberação de neurotransmissores, o aumento do fluxo sanguíneo e da vascularização cerebral e o aumento na síntese dos fatores neurotróficos, como o BDNF, que favorecem a neurogênese e a plasticidade cerebral. (COELHO et al., 2014, p. 663).

Com isso, pode-se notar que a prática de atividades físicas atua de forma benéfica no funcionamento cognitivo, tornando evidente a relação entre os níveis de atividade física e o estado de saúde mental, onde há uma menor prevalência de 
indicadores de depressão e demência para os idosos que praticam atividades físicas regularmente (BENEDETTI et al., 2008).

Além de influenciar de forma positiva em processos mentais, a prática de atividade físicatambém atua beneficamente ao tratamento da síndrome sarcopénica e consequentemente contra as quedas. A síndrome sarcopénica é definida com uma condição em que há perda da massa muscular e da força física, sendo relacionada com a idade. Portanto, o exercício físico está associado ao aumento da força muscular e a preservação do bom desempenho da motricidade, evitando assim as quedas dos idosos que podem levar até mesmo à morte (SILVA, 2012). As quedas também são um grande problema que afeta a população idosa, pois estas não se restringem apenas ao ato de cair, mas abrange outros fatores secundários como por exemplo uma fratura de fêmur, um traumatismo craniano entre outros que podem ocorrer na queda. Segundo Giles (2005 p: 45) o exercício físico pode "contribuir para a redução da fratura do fémur até 50\%”. Sendo, portanto, a atividade física um fator de grande importância para evitaressa problemática.

O processo de envelhecimento também é marcado pelo aumento do risco de eventos cardiovasculares, assim como, a prevalência da síndrome metabólica. Segundo Oliveira e colaboradores (2010), o envelhecimento cardiovascular promove Alterações na matriz proteica extracelular, mais especificamente no colágeno do coração e dos vasos sanguíneos do idoso, estas alterações levam a um aumento progressivo da rigidez pericárdica, valvular, miocárdica e vascular. Todo esse processo leva o idoso a ter mais propensões a problemas no coração como obstrução das artérias, hipertensão arterial e infarto agudo do miocárdio (IAM).

Um estudo realizado em 2017 com 1004 indivíduos, os quais foram divididos em 683 adultos (com idade entre 18 e 59 anos) e 321 idosos (idade superior a 60 anos), concluiu que aprática de atividade física por adultos e idosos, tem um efeito sobre o risco cardiovascular quando é realizada regularmente de forma moderada ou intensa (CICHOCK et al., 2017). Como benefícios ao sistema cardiovascular, a atividade física implica na redução do colesterol total (CT), lipoproteína de baixa densidade (LDL-C), triglicerídeos (TG), pressão arterial, melhora nos níveis séricos de colesterol e lipoproteína de alta densidade (HDL-C), além de contribuir para o controle glicêmico, para as respostas fisiológicas relacionadas ao aumento da 
demanda metabólica, e ao aporte de oxigênio associado aos gastos musculares (ABETE et al., 2011).

Como visto acima, a atividade física também atua no controle glicêmico e, sabe-se quegrande parte dos idosos apresentam Diabetes (mais comumente tipo 2). O diabetes mellitus (DM) é uma síndrome que constitui um problema de saúde pública, devido à elevada prevalência, morbimortalidade e custos do tratamento (RAINOR, 2001). O DM é caracterizado pela hiperglicemia, que ocorre devido à defeitos na secreção de insulina, ocasionando várias complicações como a retinopatia, que pode levar à perda da visão; nefropatia, com evolução até falência renal; neuropatia periférica, que aumenta risco de úlceras nos pés que podem desenvolver a amputação do mesmo (SILVA et al., 2018). A prática de atividade física é importante no controle glicêmico quando associada a medicação e dieta adequada, para os idosos que possuem doenças secundárias a DM, como por exemplo a retinopatia, os exercícios devem ser realizados com mais cautela, atendendo a avaliação da aptidão física e médica completas.

O efeito benéfico da prática regular de exercícios físicos vai além da atuação em depressão, controle glicêmico entre outros como já citado, estando também associado à redução dos fatores de risco cardiovasculares e à menor morbimortalidade, quando comparadas pessoasativas com inativas, o que explica a recomendação deste também na prevenção primária e no tratamento da hipertensão (FAGARD, 2006). Sabe-se que o envelhecimento está associado ao aumento da pressão arterial (PA), principalmente em relação à PA sistólica (PAS) isolada com crescente prevalência da hipertensão arterial, que promove efeitos deletérios em órgãos-alvo (SEGA et al., 2002). Paralelamente a esse processo ocorre aumento da inatividade física, entre os idosos, como demonstrado no último levantamento do Ministério da Saúde (2008), fator derisco que contribui para o aumento da incidência de doenças crônicas, entre estas a hipertensãoarterial.

Em contrapartida, a atividade física se mostra com efeitos promissores na diminuição da PA após a realização do exercício em relação aos níveis antes, sendo essa redução mais pronunciada nos indivíduos hipertensos em comparação com os normotensos (MAC DONALT, 2002). Um estudo realizado por Nogueira e colaboradores (2012), comprovou que o treinamento físico baseado em exercícios 
físicos aeróbios associados ao de resistência (circuitocom pesos) promove reduções significativas na Pressão Arterial Média (PAM) e Frequência Cardíaca (FC) de repouso. Portanto pode-se confirmar os benefícios oriundos da prática do exercício físico na redução da pressão arterial após o exercício em idosos hipertensos (Nogueiraet al., 2012).

Ressalta-se, entretanto o notável valor que o exercício físico possui na promoção da saúde, no combate ou prevenção das mais diversas doenças quer de ordem física ou psicossomática, promovendo também o bem-estar psicossocial. Desta forma concluímos que oidoso que tem um corpo sadio terá mais condições de manter uma mente sadia, elevando assim a sua qualidade de vida: "corpo são em mente sã" (SILVA, 2012).

\section{CONCLUSÃO}

Conclui-se, portanto, que a atividade física em os idosos, traz vários benefícios como por exemplo, o controle de complicações advindas de doenças crônicas, levando a uma melhoria na qualidade de vida, a uma diminuição do peso corpóreo, além do aumento da autoestima, maior disposição para o trabalho e amenização dos quadros de dor (POZENA et al., 2009). Além de combater o sedentarismo, contribui de maneira significativa para a manutenção da aptidão física do idoso, seja na sua vertente da saúde, como nas capacidades funcionais (VUORI, 1995). Outro benefício promovido pela prática de exercícios é a melhora das funções orgânicas e cognitivas, garantindo maior independência pessoal e prevenindo doenças (ANTUNES, 2006). Portanto, a prática regular de atividades físicas pelo idoso é considerada como um fator importante na promoção da saúde e na melhoria da qualidade de vida, promovendo assim, um envelhecimento saudável. 


\section{REFERÊNCIAS BIBLIOGRÁFICAS}

ABETE, I.; GOYENECHEA, E.; ZULET, M. A.; MARTINEZ, J. A. Obesity and metabolic syndrome: potential benefit from specific nutritional components. Nutr Metab CardiovascDis. $v$. 21, n. 2, p. 1-15.

ALMEIDA, Isabela Martins Franco. Influência do exercício físico na prevenção e tratamento da osteoporose: revisão bibliográfica. 2013. 25. Monografia apresentada ao Curso de Graduação em Educação Física - Universidade Federal de Minas Gerais, Minas Gerais.

ANTUNES, Hanna K. M, et al. Alterações cognitivas em idosas decorrentes do exercíciofísico sistematizado. Rev Bras Med Esporte, v. 12, n. 2, 2006.

BENEDETTI, Tânia R. Bertoldo et al. Atividade física e estado de saúde mental de idosos. Rev Saúde Pública, v. 42, n. 2, p. 302-7, 2008.

CASPERSEN, C. J.; POWELL, K. E.; CHRISTENSEN, G. M. Physical activity, exercise, and physical fitness: definitions and distinctions for health-related research. Public HealthReports, v. 100, p. $126-131,1985$.

CICHOCK, Marcelo et al. Atividade física e modulação do risco cardiovascular. Rev Bras Med Esporte, v. 23, n. 1, 2017.

COELHO, Flávia et al. Exercício Físico no Envelhecimento Saudável e Patológico: Da teoriaà prática. 1 ed.Curitiba: CRV, 2013, 462p.

COELHO, Flávia Gomes de Melo; Júnior, Jair Sindra Virtuoso. Atividade Física e SaúdeMental do Idoso. Rev Bras Ativ Fis Saúde, p. 663-664, 2014.

CRUZ, Danielle Teles; CRUZ, Felipe Moreira; RIBEIRO, Aline Lima; VEIGA, CarolineLagrotta; LEITE, Isabel Cristina Gonçalves. Associação entre capacidade cognitiva e ocorrência de quedas em idosos. Cad Saúde Coletiva. v. 23, n. 4, p. 386-93, 2015.

FAGARD, RH. Exercise is good for your blood pressure: effects of endurance training and resistance training. Clin. Exp. Pharmacol. Physiol. v. 36, n. 9, p. 853-6, 2006.

FREITAS, C. et al. Aspectos motivacionais que influenciam a adesão a manutenção de idososa programas de exercícios físicos. Rev Bras Cineantrop \& Desemp humano, v. 9, n.1, 2007.

GILES, W.H. La Activité Physique au Quotidien Protege Votre Santé. Ed INPES, Paris,2005.

GORDILHO, A. Depressão, ansiedade, outros distúrbios afetivos e suicídio. In: Freitas, E.V.et al. Tratado de Geriatria e Gerontologia. Rio de Janeiro: Guanabara Koogan, p. 204-215,2002.

GREMEAUX, V. et al. Exercise and longevity. Maturitas, v.73, 2012.

GUSMÃO, Carlos Vinícius Buarque de. BELANGERO, William Dias. Como a célula óssea reconhece o estímulo mecânico. Rev. Bras. Ortop., v. 44, p. 299-305, 2009.

HALLAL, P. et al. Tendências temporais de atividade física no Brasil (2006 -2009). Rev. Bras Epidemiologia, v.14, n.1, 2011.

KOENEMAN, M. A. et al. Determinants of physical activity and exercise in healthy olderadults: a systematic review. Int J Behav Nutr Phys Act, v. 8, p.142, 2011. 
KRITZ-SILVERSTEIN, D.; BARRETT-CONNOR, E.; CORBEAU, C. Cross-sectional and prospective study of exercise and depressed mood in the elderly: the Rancho Bernardo study. Am J Epidemiol., v. 153, n. 6, p. 596-603, 2001.

KRYGER, Meir; ROTH, Thomas; DEMENT, William. Normal human sleep: An overview. MACDONALD, Jr. Potential causes, mechanisms, and implications of post exercisehypotension. J Hum Hypertens. v. 16, p. 225-36, 2002.

MATSUDO, S. Avaliação do idoso: física e funcional. Londrina: Midiograf, 2000.

MORENO, Cláudia Roberta de Castro; SANTOS, Jair Licio Ferreira; et al. Problemas de sono em idosos estão associados sexo feminino, dor e incontinência urinária. Rev. bras. epidemiol., v. 21, n. 2, 2018.

NOGUEIRA, Ingrid Correia. Efeitos do exercício físico no controle da hipertensão arterial em idosos: uma revisão sistemática. Rev. Bras. Geriatr. Gerontol., Rio de Janeiro, v. 15, n. 3, p. 587-601, 2012.

OLIVEIRA, Mateus Silva; REZENDE, Paulo Cury; MORAES, Ricardo Casalino Sanches. Envelhecimento Cardiovascular e Doenças Cardiovasculares em Idosos. Revista MedicinaNet, 2010.

PASCHOAL, S. M. P. Qualidade de vida do idoso: construção de um instrumento de avaliação através do método do impacto clínico. [Tese de Doutorado]. São Paulo: USP; 2004.

Principles and practice of sleep medicine ( $5^{\circ}$ Ed), v. 5, p. 16-26, 2011.

RAINOR, J. Diabetes 2001. Vital Statistics. Alexandria: American Diabetes Association; 2001. p. 43-74.

SAÚDE. MDS-SDVE. Vigilância de fatores de risco e proteção para doenças crônicaspor inquérito telefônico - Vigitel. Brasília, DF, 2008.

SCOTT, A.; et al. Mechanotransduction in Human Bone In Vitro Cellular Physiology that Underpins Bone Changes with Exercise. Sports Med., v. 38, p. 139-160, 2008.

SEGA, R. et al. Blood pressure variability and organ damage in a general population: results from the Pamela study (pressioni arteriose monitorate e loro associazioni). Hypertension. v.39, p. 710-4, 2002.

SEGUIN, R. et al. Sedentary Behavior and Physical Function Decline in Older Women:Findings from the Women'Health Initiative. Journal of Aging Research, 2012.

SILVA, Kézia Katiane Medeiros; LIMA, Gleyce Any Freire; et al. Alterações do sono e a interferência na qualidade de vida no envelhecimento. Rev enferm UFPE on line. v. 11, n. 1,p. 422-428, 2017.

SILVA, Luciano Flausino. A importância do execício físico na vida do idoso. 2012. 33.Escola superior de educação João de Deus (Curso de Gerontologia Social), Lisboa.

SILVA, Marcia Regina; FERRETTI, Fátima; PINTO, Samira da Silva; FILHO, Odanor Ferretti Tombini. Sintomas depressivos em idosos e sua relação com dor crônica, doenças crônicas, qualidade do sono e nível de atividade física. BrJP. v. 1, n. 4, 2018.

SPIRDUSO, W.W. Dimensões Físicas do Envelhecimento. Ed. Manole, 2005. 482p. 
SUZMAN, Richard; BEARD, Jhon R.; BOERMA, Ties; CHATTERJI, Somnath. Health in an ageing world: what do we know? Lancet [Internet]. 2015.

TAIROVA, O. S.; DI LORENZI, D. R. S. Influência do exercício físico na qualidade de vidade mulheres na pós-menopausa: um estudo caso-controle. Revista Brasileira de Geriatria e Gerontologia. Rio de Janeiro, v. 14, n. 1, p. 135-145, 2011.

TAYLOR, A. H. Physical activity and older adults: a review of health benewts and the e Vectiveness of interventions. J Sports Sci, v. 22, p. 703-725, 2004.

THIEBAULD, C., SPRUMONT, P. O Desporto depois dos 50 anos. Ed Piaget. Lisoba, 2009 VUORI, I. Exercise and physical health musculoskeletal health and functional capabilities. Res Q Exerc Sport, v. 66, p. 276-85, 1995.

WORLD HEALTH ORGANIZATION. Assessment of fracture risk and its application toscreening of postmenopausal osteoporosis: report of a WHO Study Group. World HealthOrgan Tech Rep Ser, v. 843, p. 1-129, 1994.

WORLD HEALTH ORGANIZATION. Physical activity and older people. 2002. 